\title{
First Opioid Prescription and Subsequent High-Risk Opioid Use: a National Study of Privately Insured and Medicare Advantage Adults
}

\author{
Yongkang Zhang, $P h D^{7}$, Phyllis Johnson, $M B A^{7}$, Philip J. Jeng, $M S^{l}, M$. Carrington Reid, MD, $P h D^{2}$, \\ Lisa R. Witkin, MD ${ }^{3,4}$, Bruce R. Schackman, PhD ${ }^{1,2,5}$, Jessica S. Ancker, $P h D^{7}$, and Yuhua Bao, $P h D^{1,5}$
}

'Department of Healthcare Policy \& Research, Weill Cornell Medical College, New York, NY, USA; ${ }^{2}$ Department of Medicine, Weill Cornell Medical College, New York, NY, USA; ${ }^{3}$ Department of Anesthesiology, Weill Cornell Medical College, New York, NY, USA; ${ }^{4}$ Division of Pain Medicine, New York-Presbyterian/Lower Manhattan Hospital, New York, NY, USA; ${ }^{5}$ Department of Psychiatry, Weill Cornell Medical College, New York, NY, USA.

\begin{abstract}
BACKGROUND: National guidelines make recommendations regarding the initial opioid prescriptions, but most of the supporting evidence is from the initial episode of care, not the first prescription.

OBJECTIVE: To examine associations between features of the first opioid prescription and high-risk opioid use in the 18 months following the first prescription.

DESIGN: Retrospective cohort study using data from a large commercial insurance claims database for 20112014 to identify individuals with no recent use of opioids and follow them for 18 months after the first opioid prescription.
\end{abstract}

PARTICIPANTS: Privately insured patients aged 18-64 and Medicare Advantage patients aged 65 or older who filled a first opioid prescription between 07/01/2011 and 06/30/2013.

MAIN OUTCOMES AND MEASURES: High-risk opioid use was measured by having (1) opioid prescriptions overlapping for 7 days or more, (2) opioid and benzodiazepine prescriptions overlapping for 7 days or more, (3) three or more prescribers of opioids, and (4) a daily dosage exceeding 120 morphine milligram equivalents, in each of the six quarters following the first prescription.

KEY RESULTS: All three features of the first prescription were strongly associated with high-risk use. For example, among privately insured patients, receiving a long- (vs. short-) acting first opioid was associated with a 16.9-percentage-point increase (95\% CI, 14.3-19.5), a daily MME of 50 or more (vs. less than 30 ) was associated with a 12.5percentage-point increase (95\% CI, 12.1-12.9), and a supply exceeding 7 days (vs. 3 or fewer days) was associated with a 4.8-percentage-point increase (95\% CI, 4.55.2 ), in the probability of having a daily dosage of 120 MMEs or more in the long term, compared to a sample mean of $4.2 \%$. Results for the Medicare Advantage patients were similar.

CONCLUSIONS: Long-acting formulation, high daily dosage, and longer duration of the first opioid prescription

Electronic supplementary material The online version of this article (https://doi.org/10.1007/s11606-018-4628-y) contains supplementary material, which is available to authorized users.

Received February 5, 2018

Revised May 24, 2018

Accepted July 27, 2018

Published online September 11, 2018 were each associated with increased high-risk use of opioids in the long term.

KEY WORDS: prescription drug abuse; pain; health services research; physician behavior.

J Gen Intern Med 33(12):2156-62 DOI: $10.1007 / \mathrm{s} 11606-018-4628-y$

(C) Society of General Internal Medicine 2018

\section{INTRODUCTION}

From 1999 to 2014, prescription opioid overdoses accounted for more than 193,000 deaths in the USA. ${ }^{1}$ This epidemic was paralleled by a tripling of the amount of opioids (in terms of morphine milligram equivalents or MMEs) received per capita over 1999-2015. ${ }^{2}$ While opioid therapies were frequently initiated for acute pain, $6 \%$ of patients newly prescribed opioids continued to use opioids at 1 year and $2.9 \%$ continued to use at 3 years, ${ }^{3}$ despite lack of evidence of clinical efficacy and potential risks for opioid misuse and opioid use disorder. ${ }^{4}$ There is thus heightened scrutiny of initial exposure to prescription opioids. Recent policies and practices in this area include the release of the Guideline for Opioid Prescribing for Chronic Pain by the Centers for Disease Control and Prevention (CDC) ${ }^{5,6}$ state legislations limiting the initial amount of opioids prescribed to patients, ${ }^{7}$ as well as various strategies used by health insurance plans, prescription benefit management companies, and chain pharmacies to limit initial opioid prescribing.

Evidence is accumulating that the amount of initial opioid exposure is linked to long-term use. The strongest evidence so far pertains to the initial episode of opioid therapy (variably defined in the literature), rather than the initial prescription. For example, one study ${ }^{8}$ found that the number of prescription fills and the cumulative MMEs dispensed during 30 days after opioid initiation were associated with long-term use. Another study ${ }^{3}$ defined the first episode of opioid use as continuous use of opioids with a gap of no greater than 30 days and found similar associations. A more recent study ${ }^{9}$ examined newly initiated opioid use post-surgery and found that incremental number of prescription fills and duration of continuous use 
were associated with increased probability of medical events suggesting opioid misuse. The care episodes in these studies likely involved multiple prescribers who may or may not have communicated with one another. Evidence is limited on how prescribing decisions regarding the first opioid prescriptions, which are well within the control of the prescribing clinician, are associated with opioid use in the long-term that puts patients at heightened risks for misuse and overdose ("highrisk use" hereafter). One exception ${ }^{10}$ is a cohort study of veterans who had chronic painful conditions and started opioid therapy. The study focused on the duration of action of the first opioid and found that long-acting status was associated with a twice as high risk of unintentional overdose injury.

In this study, we used data from a large commercial insurance claims database to address this knowledge gap. Our study adds to the existing literature by focusing on the first opioid prescriptions, by examining three salient features of the first prescriptions highlighted in the CDC Guideline (duration of action, dosage, and days of supply), ${ }^{5,6}$ and by examining a comprehensive set of outcomes pertaining to high-risk use of opioids in the long term. The CDC Guideline recommends using immediate-release/short-acting opioids and avoiding extended release/long-acting drugs (recommendation 4). In addition, the Guideline recommends using the lowest effective dosage and evaluating opioid-related benefits and risks when increasing dosages above 50 MMEs per day and carefully justifying an increase in dose above 90 MMEs per day (recommendation 5). The Guideline also recommends restricting the quantity of opioids prescribed to the expected duration of pain severe enough to require opioids (e.g., 3 days for acute pain) (recommendation 6). We conducted separate analyses for privately insured adults 18-64 and for older adults (65+) insured by Medicare Advantage plans. We hypothesized that long- (vs short-) acting opioid formulations, higher daily dosage, and increased number of days of supply would all be independently associated with an increased risk of high-risk opioid use in the 18 months following the initial opioid prescription.

\section{METHODS}

\section{Data}

We used data from the 2011-2014 Health Care Cost Institute (HCCI) insurance claims database, ${ }^{11}$ including claims from private insurance plans (both employer sponsored and individual-market based) and Medicare Advantage plans offered by Aetna, Humana, and UnitedHealthcare. The HCCI data include beneficiary enrollment information, inpatient facility claims, outpatient facility claims, physician claims, and pharmacy claims. Pharmacy claims provide information on the National Drug Code (NDC), days of supply, quantity and unit, and strength/dosage for each prescription. In addition, we extracted ZIP code-level sociodemographic profiles of residents from the American Community Survey ${ }^{12}$ and merged these data with the HCCI claims based on patient residential ZIP code. Recent evidence indicates that certain community characteristics are associated with long-term and high-risk prescription opioid use. ${ }^{13}$

\section{Study Population and Sample}

Our study population consisted of privately insured adults aged 18 to 64 and Medicare Advantage (MA) patients 65 or older who did not have recent use of prescription opioids (defined with a "look-back" period of 6 months without any opioid prescription) and filled a first opioid prescription between $07 / 01 / 2011$ and $06 / 30 / 2013$. To be included in the sample, patients had to have at least 6 months of continuous enrollment in a private health plan or a MA plan contributing data to HCCI prior to the month of their first opioid prescription (the "index month") and be continuously enrolled for at least 18 months after the index month. We excluded patients with a cancer diagnosis in the 6 months prior to the index month because of our focus on individuals with non-cancer pain. Separate analyses were conducted for the non-elderly privately insured sample and the elderly MA sample. Patients who transitioned from one type of insurance to another (e.g., from an employer sponsored plan to an MA plan; $N=256$ ) accounted for only $0.05 \%$ of the original sample and were too few to warrant a separate analysis. We excluded them from the analysis.

\section{Features of the First Opioid Prescription}

We focused on duration of action, dosage, and days of supply of the first opioid prescription, consistent with recommendations made in the 2016 CDC Guideline. ${ }^{5,}{ }^{6}$ Using a CDC database linking NDCs with drug characteristics, ${ }^{14}$ we (1) determined the long- vs. short-acting status of the first prescription and (2) calculated daily MMEs (= strength per unit $\times$ (\# of units/days of supply) $\times$ MME conversion factor ${ }^{14}$ ). In the case of multiple opioids filled on the same day, we determined the index prescription to be long-acting as long as one of the prescriptions was long-acting. We further categorized daily MMEs into three levels: $<30, \geq 30$ but $<50$, or $\geq 50$; we did not use 90 MMEs as a cut-point because only $7 \%$ of our sample were prescribed a daily MME of 90 or more at their first prescriptions, but conducted a sensitivity analysis with the cut-points of 50 and 90 MMEs. We categorized days of supply into $\leq 3$ days, $4-7$ days, and $>7$ days.

\section{Outcome Measures}

We measured high-risk opioid use over each of the six 3month intervals ("quarters" hereafter) following the index month, consistent with previous studies. ${ }^{15,16}$ We included a patient-quarter in the analysis if the patient had prescription opioids for at least 1 day in that quarter. We defined four dichotomous indicators of high-risk opioid use based on a literature review: (1) having opioid prescriptions overlapping 
for 7 days or more, ${ }^{16,17}$ (2) having opioid and benzodiazepine prescriptions overlapping for 7 days or more, ${ }^{18-20}$ (3) having filled opioid prescriptions from multiple prescribers, ${ }^{21}$ and (4) having a daily average MMEs exceeding $120 .^{15,20,22}$ Previous studies differ in the cut-points to dichotomize multiple prescribers to capture potential "doctor shopping"; they also differ in the time interval used to assess multiple prescribers. ${ }^{21-23}$ We examined the outcome of three or more prescribers over each 3-month interval.

\section{Statistical Analyses}

We estimated a model for each of the four high-risk opioid use outcomes. The unit of analysis was patient-quarter with at least 1 day of opioid use. Patients contributed up to six quarters to the analysis. All models controlled for ordinal indicators of the quarters (second, third, sixth, with the first quarter as the reference); calendar year indicators; patient demographics (age groups, sex); dichotomous indicators of back pain, neck pain, arthritis/joint pain, and other pain (based on ICD-9 diagnostic codes shown in Online Appendix 1); an indicator of any mental health disorder; and dichotomous indicators of any alcohol use disorder, any drug use disorder, and tobacco use disorder (Online Appendix 2). We also controlled for socio-demographic profiles at the patient's residential ZIP code, including percentage of residents who were nonHispanic black, who were Hispanic, who had some college, who were unemployed, who were living in poverty, percentage of households led by married couples, and urban/rural status of ZIP code based on Census Bureau definitions. ${ }^{24}$

Although not the focus of our study, we also examined how features of the first prescription were associated with some opioid use in each of the six quarters following the index month by estimating a similar regression model as in the analysis of high-risk use, but using the samples of all patients who initiated opioids.

We estimated both a linear probability model and a logistic model for each analysis. Results in terms of incremental probabilities associated with the features of the first opioid prescription were almost identical between the two modeling approaches. We thus present our results below based on the linear probability models. All analyses were conducted using Stata version $14 .^{25}$ We generated robust standard errors by taking into account clustering of quarters pertaining to the same patient. ${ }^{26}$

This study was approved by the Institutional Review Board of the Weill Cornell Medical College.

\section{RESULTS}

A total of 403,664 non-elderly, privately insured patients and 107,509 elderly MA patients initiated opioid therapy between 07/01/2011 and 06/30/2013. Among these patients, 196,375 non-elderly, privately insured patients and 63,419 elderly MA patients contributed at least one quarter (subsequent to the index month) to the analysis of high-risk use (Table 1). Fewer than $1 \%$ of patients in both samples were prescribed a longacting opioid at the first prescription. For $26.7 \%$ of the privately insured patients and $19.3 \%$ of the MA patients, average daily dosage of the first prescription was $50 \mathrm{MMEs}$ or higher. More than $18 \%$ of privately insured patients and more than $35 \%$ of MA patients received more than 7 days of supply at their first opioid prescriptions.

\section{First Opioid Prescription and High-Risk Opioid Use}

In the non-elderly privately insured sample, of the 340,629 patient-quarters with at least 1 day of opioid use, $4.0 \%$ had overlapping opioid prescriptions, $6.7 \%$ had overlapping opioid-benzodiazepine prescriptions, $2.5 \%$ had three or more prescribers, and $4.2 \%$ had 120 or more daily average MMEs. Of the 133,444 patient-quarters in the elderly MA sample, prevalence of the four high-risk outcomes was $5.3 \%, 8.1 \%$, $2.3 \%$, and $1.4 \%$, respectively. We report below regressionadjusted results pertaining to the privately insured sample (regression outputs in Online Appendix 3). Results pertaining to the MA sample were very similar and presented in Online Appendix 5.

All three features of the first prescription were associated with the probability of having opioids overlapping for at least 7 days in the six quarters following the index month (Fig. 1). In particular, receiving a long- (vs. short-) acting opioid was associated with a 15-percentage-point (more than fourfold) increase (95\% Confidence Interval or CI, 12.7 to 16.7 percentage points or pps, $p<0.001)$. A daily dosage of 50 MMEs or more was associated with a slightly increased probability compared to the two lower categories $(p<0.001)$. Receipt of more than 7 days of opioids was associated with a 7 percentage-point increase $(95 \% \mathrm{CI}, 6.2$ to $7.0 \mathrm{pps} ; p<0.001)$ and a 6-percentage-point increase (95\% CI, 5.3 to $6.0 \mathrm{pps}$; $p<0.001)$ compared to a supply of 3 or fewer days and of 4 7 days, respectively. A similar pattern was observed for the outcome of overlapping opioids and benzodiazepines (Fig. 2). Both long-acting (vs. short-acting) status (5.8 pps; 95\% CI, 3.5 to $8.0 \mathrm{pps} ; p<0.001$ ) and more-than-7-day-supply (vs. 3 or fewer days) (8.7 pps; $95 \% \mathrm{CI}, 8.2$ to $9.2 \mathrm{pps} ; p<0.001$ ) were associated with more than doubling of the probability of opioid-benzodiazepine overlap.

Long- (vs. short-) acting status of the first prescription was associated with a 2-percentage-point increase (95\% CI, 0.9 to $2.7 \mathrm{pps} ; p<0.001)$ in the probability of having three or more prescribers of opioids in a quarter. A daily dosage of $50 \mathrm{MMEs}$ or more (vs. lower dosages) and a more-than-7-day supply (vs. fewer days) were associated with a slight increase in the probability of this outcome (Fig. 3).

All three features of the first prescription were strongly associated with a daily dosage of $120 \mathrm{MME}$ or more in the long term (Fig. 4). Specifically, long- (vs. short-) acting status was associated with a 16.9-percentage-point increase $(95 \% \mathrm{CI}$, 
Table 1 Demographic and Clinical Characteristics of Patients Included in the Study Samples and Features of the First Opioid Prescriptions

\begin{tabular}{lll}
\hline \hline Characteristics & $\begin{array}{l}\text { Private insurance, } \\
18-64 \text { years of age }\end{array}$ & $\begin{array}{l}\text { Medicare advantage, } \\
65 \text { and older }\end{array}$ \\
& & $N=63,419$ \\
\hline$\%$ & $\%$ \\
\hline
\end{tabular}

Patient demographic characteristics and health conditions

Age

$18-34$

$35-44$

$45-54$

$55-64$

$65+$

Female

$35.5 \%$

$23.4 \%$

$24.3 \%$

$16.9 \%$

$55.3 \%$

$11.4 \%$

$5.3 \%$

Neck pain

Arthritis/joint pain

Other pain

$23.0 \%$

$12.8 \%$

$12.6 \%$

Any mental health

Any alcohol use disorder

Any drug use disorder

$0.6 \%$

$0.5 \%$

$2.2 \%$

Socio-demographic profiles at patient residential ZIP code*

$\%$ of residents that were non-Hispanic black

1 st quartile

$1.8 \%$

$11.0 \%$

$41.7 \%$

$45.5 \%$

3rd quartile

4th quartile

$\%$ of residents that were Hispanic
1 st quartile

2nd quartile

3rd quartile

$16.0 \%$

$32.3 \%$

$49.6 \%$

$\%$ of residents

who had some college education

1st quartile

2nd quartile

3rd quartile

4th quartile

$8.2 \%$

$16.6 \%$

$25.6 \%$

$49.6 \%$

$100.0 \%$

$59.1 \%$

$17.6 \%$

$5.5 \%$

$46.0 \%$

$16.8 \%$

$12.0 \%$

$0.6 \%$

$0.2 \%$

$\%$ of reside

1st quartile

2nd quartile

3rd quartile

4th quartile

$\%$ of residen

2nd quartile

3rd quartile

4th quartile

$\%$ of househ

1st quartile

2nd quartile

3rd quartile

4th quartile

ZIP code having no

older who

$11.5 \%$
$38.3 \%$

$33.2 \%$

$17.0 \%$

$3.1 \%$

$14.6 \%$

$38.1 \%$

$44.2 \%$

$3.6 \%$

$21.3 \%$

$36.1 \%$

$38.9 \%$

$12.7 \%$

$25.6 \%$

$29.9 \%$

$31.8 \%$

urban-area

Features of the first opioid prescription

Long-acting vs shorting acting

\begin{tabular}{lll}
$\begin{array}{l}\text { Long-acting } \\
\text { Short-acting }\end{array}$ & $0.9 \%$ & $1.0 \%$ \\
Daily MMEs & $99.1 \%$ & $99.3 \%$ \\
$<30$ & $32.2 \%$ & \\
$30-50$ & $41.2 \%$ & $43.2 \%$ \\
$\geq 50$ & $26.7 \%$ & $37.6 \%$ \\
Days of supply & & $19.3 \%$ \\
$\leq 3$ & $40.2 \%$ & \\
$4-7$ & $41.6 \%$ & $26.3 \%$ \\
$>7$ & $18.3 \%$ & $38.2 \%$ \\
\hline
\end{tabular}

Notes: Samples include patients who initiated opioid therapy between 07/ $01 / 2011$ and 06/30/2013 (with no opioid use in the 6 months prior to initiation) and who had some opioid use in at least one of six quarters after the month of opioid initiation. MMEs morphine milligram equivalents * Percentages presented are \% of sample patients who resided in a ZIP code that belonged to the first, second, third, or fourth quartile of all residential ZIP codes in the USA for a given socioeconomic profile
0.200

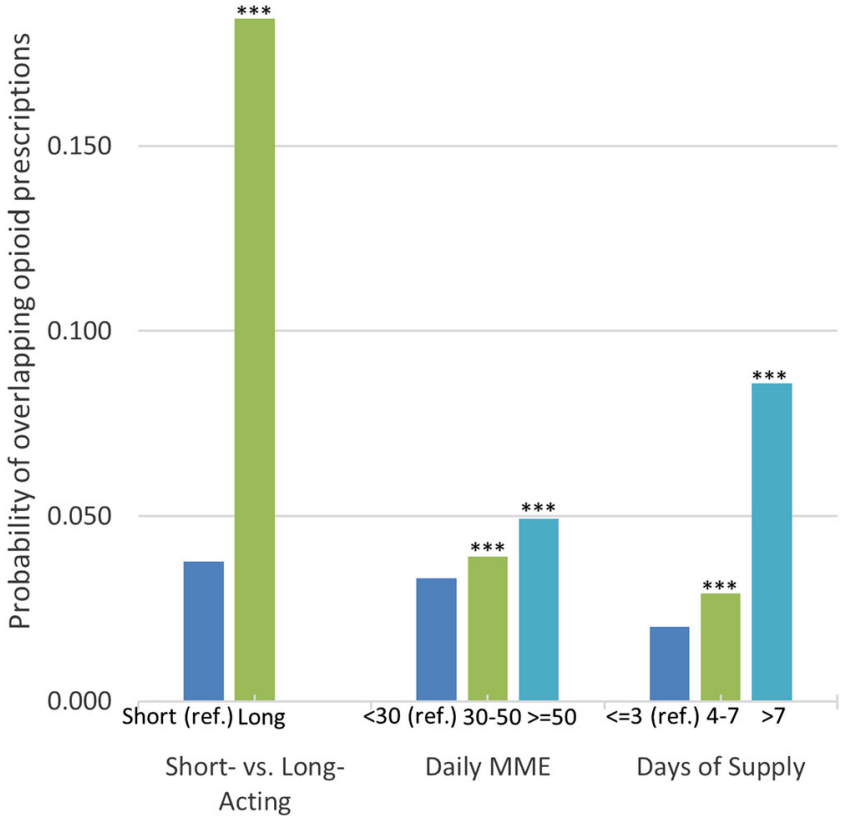

Fig. 1 Predicted probability of overlapping opioid prescriptions by features of the first opioid prescription, privately insured patients

18-64 years of age. Notes: significance denotes the difference between reference category and other categories; MME, morphine milligram equivalent; ref., reference group; ${ }^{* * *} p<0.001$; ${ }^{* *} p<0.01$; $* p<0.05$.

14.0 to $19.5 \mathrm{pps} ; p<0.001)$ and a daily MME of 50 or more (vs. less than $30 \mathrm{MMEs}$ ) was associated with a 12.5 -percentage-point increase (95\% CI, 12.1 to $12.9 \mathrm{pps} ; p<0.001)$-a

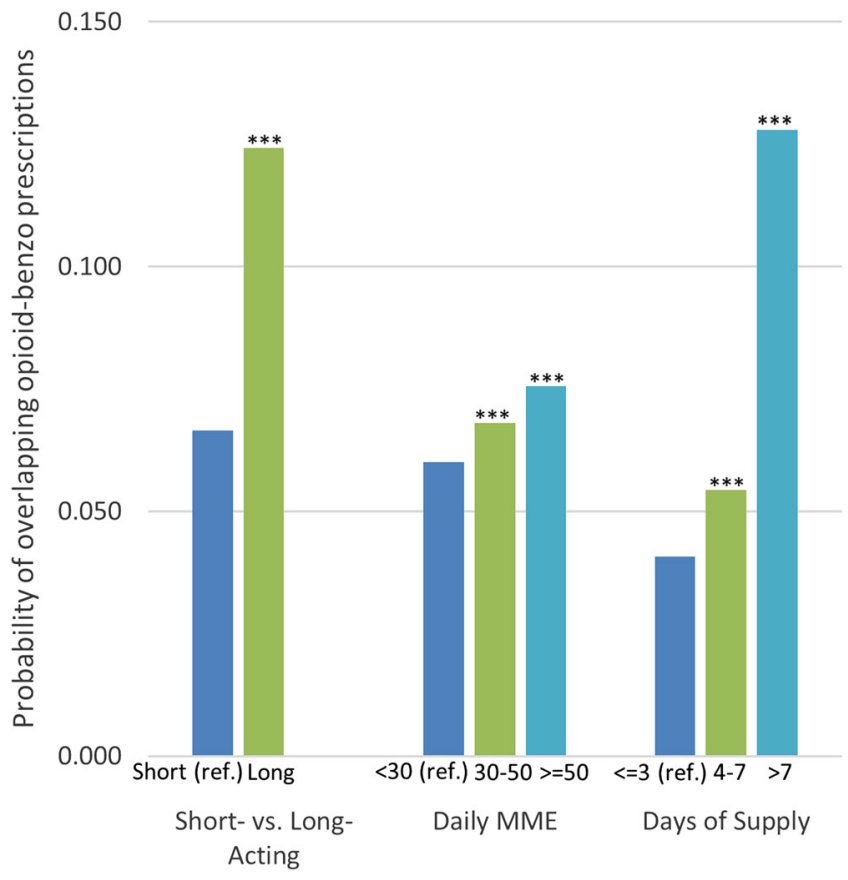

Fig. 2 Predicted probability of overlapping opioids and benzodiazepines by features of the first opioid prescription, privately insured patients 18-64 years of age. Notes: significance denotes the difference between reference category and other categories; MME morphine milligram equivalent; ref., reference group; $* * * p<0.001$; $* * p<0.01 ; * p<0.05$. 
0.050

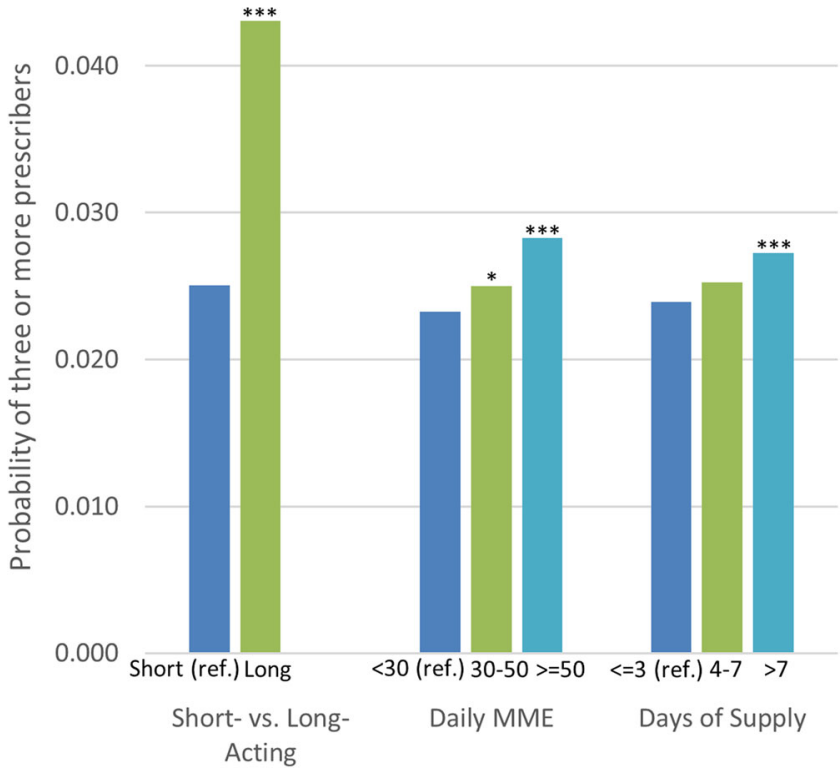

Fig. 3 Predicted probability of three or more prescribers of opioids by features of the first opioid prescription, privately insured patients

18-64 years of age. Notes: significance denotes the difference between reference category and other categories; MME, morphine milligram equivalent; ref., reference group; $* * * p<0.001 ; * * p<0.01$; $* p<0.05$.

three- to fivefold increase - in the predicted probability of this outcome. A more-than-7-day supply (vs. 3 or fewer days) was associated with a 4.8 pps of increase (95\% CI: 4.5 to 5.2).

0.250

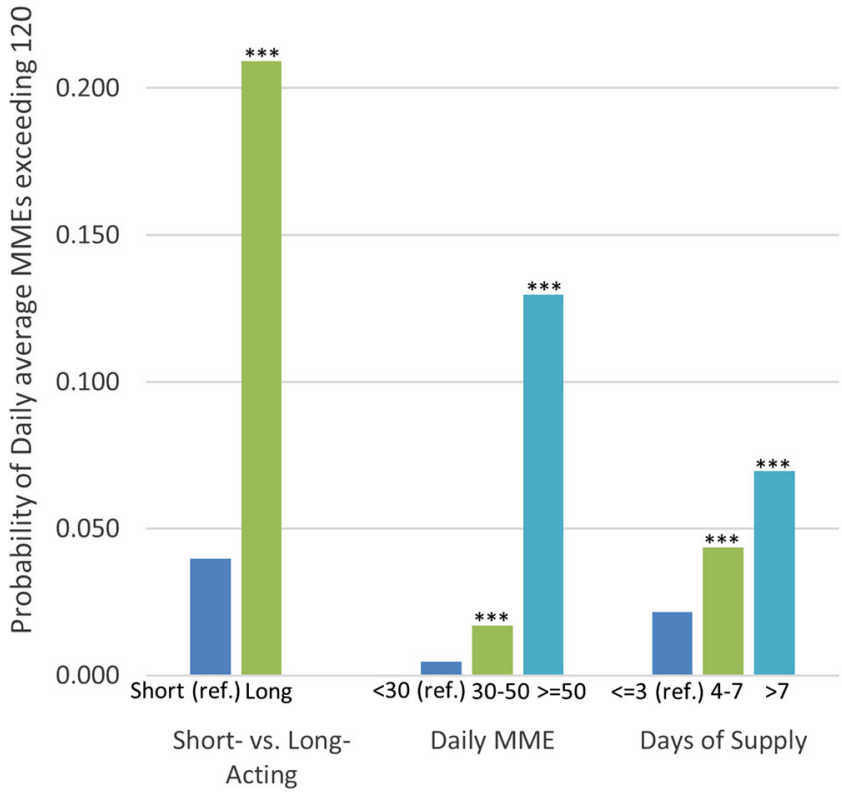

Fig. 4 Predicted probability of 120 or more daily average MMEs by features of the first opioid prescription, privately insured patients 18-64 years of age. Notes: significance denotes the difference between reference category and other categories; MME, morphine milligram equivalent; ref., reference group; $* * * p<0.001$; $* * p<0.01$; $* p<0.05$.
Using 50 and 90 daily MMEs as cut-points in categorizing the dosage of the first prescription generated results suggesting similar or stronger associations (Online Appendix 4).

\section{First Opioid Prescription and Opioid Use in the Long Term}

Based on this analysis, we found that, in the privately insured sample, a long-acting opioid at the first prescription was associated with an 8-percentage-point increase in the probability of some opioid use (95\% CI, 6.4 to $9.1 \mathrm{pps} ; p<0.001$ ); having received 7 days or more of opioids was associated with an increase of 15 pps (95\% CI, 15.0 to $15.6 ; p<0.001$ ), compared to a supply of 3 or fewer days, and an increase of 13 pps (95\% CI, 12.9 to $13.4 ; p<0.001$ ), compared to a supply of 4-7 days (Online Appendix 6). Results for the MA sample were very similar (Online Appendix 7).

\section{DISCUSSION}

Using population data, we found that each of the three features of the first opioid prescriptions addressed in the 2016 CDC Guideline (duration of action, dosage, and days of supply) was strongly and independently associated with high-risk opioid use over the 18 months following the index prescription month. Our study adds to the existing literature by focusing on characteristics of the first opioid prescriptions, by examining all three salient features of the first prescriptions specifically addressed in the 2016 CDC Guideline, and by examining a comprehensive set of outcomes pertaining to high-risk opioid use in the long term. Additional strengths of our study include the use of a large national commercial insurance claims database covering both privately insured and Medicare Advantage adults and implications applicable to all clinical settings.

It is worth noting that long-acting opioids only accounted for $0.9 \%$ of all the first opioid prescriptions among privately insured patients and $1.0 \%$ of the elderly MA patients included in our analysis. In addition, about half of all patients who filled a long-acting opioid also filled a short-acting opioid on the same day. Patients prescribed long-acting opioids may have continuous pain that short-acting opioids alone may not effectively alleviate, ${ }^{27}$ although recent guidelines recommend against using long-acting opioids when opioid therapy is initiated. ${ }^{6,27}$ In both of our samples, patients who received a long-acting opioid as their first prescription were 2-3 times as likely to have had back pain, neck pain, and arthritis-related pain, and to have had diagnosed mental health disorders and substance use disorders in the 6 months prior to the first opioid prescription. Future studies employing more nuanced clinical data should examine the context of long-acting opioid prescribing when initiating opioid therapy and evaluate associated benefits and risks.

Our findings regarding the heightened risks associated with more than 7 days of opioids at the first prescription are 
meaningful at the population level given the large number of patients (18\% among the privately insured and 36\% among the MA patients) who received a first prescription exceeding 7 days in duration. These findings support the recent wave of policies to limit the duration of the first opioid prescriptions, including state laws or agency rules to limit the amount of opioids prescribed at the first prescription, usually in terms of days of supply or total morphine milligram equivalents, ${ }^{7}$ pharmacy management strategies adopted or planned by state Medicaid programs to implement the CDC Guideline, private health plan coverage limitations, ${ }^{28}$ and, more recently, policies adopted by chain pharmacies. ${ }^{29}$ Implementation of these policies, however, may create important barriers to effective pain management for patients who need continued opioid therapy beyond 7 days and/or who have limited means to access alternative pain management strategies. For example, time, transportation, and co-payment associated with follow-up doctor visits and visits to the pharmacy to obtain continued opioid prescriptions may be overwhelming to some patients, such as older adults with mobility issues. Currently, very few jurisdictions have adopted legislation or rules to address these barriers. ${ }^{30}$ In addition, while these policies are meant to apply to the initial opioid prescriptions only, it is unknown whether they have been inadvertently applied to continuing opioid therapies, which may result in disruption in pain management and uncontrolled pain.

Our findings regarding the risks associated with a high daily MME of the first prescription suggest the need for decision support to prescribing clinicians at the point of care. In particular, electronic health record (EHR) systems could provide automatic conversion of dosages into MMEs for each opioid and/or alert prescribers of high MMEs (e.g., > = 50 MMEs) of the first prescriptions. More generally, "nudges" and other behavioral interventions built into the EHRs may be especially effective in changing prescribing behaviors without disrupting clinical work flow or infringing on clinician autonomy. Examples of such interventions include re-programming order sets to default to prescribing decisions concordant with the guidelines, ${ }^{31}$ asking prescribers to provide justification for prescriptions deemed inappropriate or inconsistent with guidelines, ${ }^{32}$ and providing prescriber-specific summary data (e.g., $\%$ of all the first opioid prescriptions with more than 7 days of supply) in comparison with peers.

Our study has a few limitations. First, as is the case for all studies using claims data, it is possible that our sample included patients who had used opioids in the past but had a recent gap in opioid use of at least 6 months. Second, the daily MMEs calculated in this study reflected the amount of opioids available to the patient rather than the actual dosages taken; this limitation is especially salient for PRN medications. Third, our data do not capture prescriptions paid for in cash. To the extent that patients whose first prescriptions were long acting, of higher dosage, or longer duration were more likely to use cash payment for their opioid prescriptions in the long term, our results would underestimate the associations between features of the first prescriptions and high-risk use in the long term. Fourth, prescriber IDs in our data had a high missing rate; there was also inconsistency in provider IDs between the pharmacy and medical claims. We were thus not able to pinpoint the healthcare setting responsible for the first prescription. Finally, features of the first opioid prescription may be correlated with unobserved and unmeasured severity or chronicity of pain. Thus, our results may be subject to remaining confounding and are best interpreted as associative rather than causal.

\section{CONCLUSIONS}

This study provides evidence that long-acting formulation, high daily dosage, and a duration exceeding 7 days of the first opioid prescription are each associated with increased highrisk use of opioids in the long term. Our findings thus provide support to policies, clinical guidelines, and health care system interventions aimed to limit prescribing of the first opioids with these features.

Corresponding Author: Yuhua Bao, PhD; Department of Healthcare Policy \& Research Weill Cornell Medical College, New York, NY, USA (e-mail: yub2003@med.cornell.edu).

Funding Information This study was funded by the Center for Health Economics of Treatment Interventions for Substance Use Disorder, HCV, and HIV (CHERISH), a National Institute on Drug Abuse Center of Excellence (Grant No. P3ODA040500; YB, PJJ, BRS), the National Institute of Mental Health (Grant No. R01MH1O420O; YB, PJ, PJJ), the National Institute on Aging (Grant Nos. P30AG022845, K24AG053462; MCR), the New York State Health Foundation (Grant No. 17-05047; JSA), the Agency for Healthcare Research and Quality (Grant No. K01HSO21531; JSA), and the Weill Cornell Medical College Department of Healthcare Policy and Research (for access to HCCI data).

\section{Compliance with Ethical Standards:}

This study was approved by the Institutional Review Board of the Weill Cornell Medical College.

Conflict of Interest: The authors declare that they do not have a conflict of interest.

\section{REFERENCES}

1. Centers for Disease Control and Prevention, National Center for Health Statistics. Number and age-adjusted rates of drug-poisoning deaths involving opioid analgesics and heroin: United States, 2000-2014. National Vital Statistics System, Mortality File https://www.cdc.gov/ nchs/data/health_policy/AADR_drug_poisoning_involving_OA_Heroin_ US_2000-2014.pdf. Accessed July 13, 2018.

2. Guy GP, Jr., Zhang $\mathbf{K}$, Bohm MK, et al. Vital signs: changes in opioid prescribing in the United States, 2006-2015. MMWR Morb Mortal Wkly Rep. 2017;66(26):697-704.

3. Shah A, Hayes CJ, Martin BC. Characteristics of initial prescription episodes and likelihood of long-term opioid use - United States, 20062015. MMWR Morb Mortal Wkly Rep. 2017;66(10):265-269.

4. Chou R, Turner JA, Devine EB, et al. The effectiveness and risks of long-term opioid therapy for chronic pain: a systematic review for a National Institutes of Health Pathways to Prevention Workshop. Ann Intern Med. 2015;162(4):276-286. 
5. Dowell D, Haegerich TM, Chou R. CDC guideline for prescribing opioids for chronic pain-United States, 2016. JAMA. 2016;315(15):1624-1645.

6. Dowell D, Haegerich TM, Chou R. CDC guideline for prescribing opioids for chronic pain - United States, 2016. MMWR Recomm Rep. 2016;65(1): 1-49.

7. Baker-White A. A look at state legislation limiting opioid prescriptions. 2017; http://www.astho.org/StatePublicHealth/A-Look-at-State-Legislation-Limiting-Opioid-Prescriptions/2-23-17/. Accessed July 13, 2018.

8. Deyo RA, Hallvik SE, Hildebran C, et al. Association between initial opioid prescribing patterns and subsequent long-term use among opioidnaïve patients: a statewide retrospective cohort study. $J$ Gen Intern Med. 2017;32(1):21-27.

9. Brat GA, Agniel D, Beam A, et al. Postsurgical prescriptions for opioid naive patients and association with overdose and misuse: retrospective cohort study. BMJ. 2018;360:j5790.

10. Miller M, Barber CW, Leatherman S, et al. Prescription opioid duration of action and the risk of unintentional overdose among patients receiving opioid therapy. JAMA Intern Med. 2015; 175(4):608-615.

11. Health Care Cost Institute. 2017; http://www.healthcostinstitute.org/. Accessed July 13, 2018.

12. United States Census Bureau. American Community Survey. https:// www.census.gov/programs-surveys/acs/. Accessed July 13, 2018.

13. Hollingsworth A, Ruhm CJ, Simon K. Macroeconomic conditions and opioid abuse. NBER Work Pap Ser. 2017;No. 23192.

14. National Center for Injury Prevention and Control. CDC compilation of benzodiazepines, muscle relaxants, stimulants, zolpidem, and opioid analgesics with oral morphine milligram equivalent conversion factors, 2016 version. 2016; http://www.pdmpassist.org/pdf/BJA_performance_ measure_aid_MME_conversion.pdf. Accessed July 13, 2018.

15. Meara E, Horwitz JR, Powell W, et al. State legal restrictions and prescription-opioid use among disabled adults. $N$ Engl $\mathrm{J}$ Med. 2016;375(1):44-53.

16. Prescription Drug Monitoring Program Training and Technical Assistance Center. DEFINITIONS OF PBSS MEASURES. http://www.pdmpassist. org/pdf/COE_documents/Add_to_TTAC/Definitions\%20of\%20PBSS \%20Measures.pdf. Accessed July 13, 2018.

17. Seal KH, Shi Y, Cohen G, et al. Association of mental health disorders with prescription opioids and high-risk opioid use in US veterans of Iraq and Afghanistan. JAMA. 2012;307(9):940-947.

18. Sun EC, Dixit A, Humphreys K, et al. Association between concurrent use of prescription opioids and benzodiazepines and overdose: retrospective analysis. BMJ. 2017;356:j760.
19. Hawkins EJ, Malte CA, Grossbard JR, Saxon AJ. Prevalence and Trends of Concurrent Opioid Analgesic and Benzodiazepine Use Among Veterans Affairs Patients with Post-traumatic Stress Disorder, 20032011. Pain Med. 2015;16(10): 1943-1954.

20. Braden JB, Russo J, Fan MY, et al. Emergency department visits among recipients of chronic opioid therapy. Arch Intern Med. 2010;170(16):1425-1432.

21. Wilsey BL, Fishman SM, Gilson AM, et al. Profiling multiple provider prescribing of opioids, benzodiazepines, stimulants, and anorectics. Drug Alcohol Depend. 2010;112(1):99-106.

22. Baumblatt $\mathbf{J}$, Wiedeman $\mathbf{C}$, Dunn $\mathbf{J} \mathbf{R}$, et al. High-risk use by patients prescribed opioids for pain and its role in overdose deaths. JAMA Intern Med. 2014;174(5):796-801.

23. Jena AB, Goldman D, Weaver L, Karaca-Mandic P. Opioid prescribing by multiple providers in Medicare: retrospective observational study of insurance claims. BMJ 2014;348:g1393.

24. United States Census Bureau. 2010 Urban Area to ZIP Code Tabulation Area (ZCTA) Relationship File. https://www2.census.gov/geo/docs/ maps-data/data/rel/ua_zcta_rel_10.txt. Accessed July 13, 2018.

25. Stata Statistical Software: Release 14 [computer program]. College Station, TX: StataCorp LP; 2015.

26. White $\mathbf{H}$. A heteroskedasticity-consistent convariance matrix estimator and a direct test for heteroskedasticity. Econometrica. 1980;48:817-830.

27. Rauck RL. What is the case for prescribing long-acting opioids over short-acting opioids for patients with chronic pain? A critical review. Pain Pract. 2009;9(6):468-479.

28. MacQuarrie B. Blue Cross cuts back on painkiller prescriptions: An 18month effort targets opiate abuse. 2014; https://search.proquest.com/ docview/1513291021/fulltext/97C2A93FA6BD4AF1PQ/1? accountid=170463. Accessed July 13, 2018.

29. Scutti S, Kounang N. CVS will limit opioid prescriptions to 7 days. 2017; http://www.cnn.com/2017/09/22/health/cvs-prescription-restrictionsopioids-bn/index.html. Accessed July 13, 2018.

30. Johnson L. Insurance Circular Letter No. 6 (2016) - Coverage for Substance Use Disorder Treatment. 2016; http://www.dfs.ny.gov/insurance/circltr/2016/cl2016_06.htm. Accessed July 13, 2018.

31. Delgado MK, Shofer FS, Patel MS, et al. Association between electronic medical record implementation of default opioid prescription quantities and prescribing behavior in two emergency departments. J Gen intern Med 2018;33:409.

32. Meeker D, Linder JA, Fox CR, et al. Effect of behavioral interventions on inappropriate antibiotic prescribing among primary care practices: a randomized clinical trial. JAMA. 2016;315(6):562-570. 